Pacific Journal of Mathematics

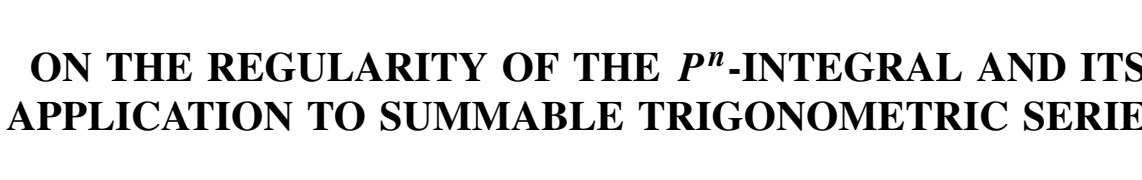




\title{
ON THE REGULARITY OF THE $P^{n}$-INTEGRAL AND ITS APPLICATION TO SUMMABLE TRIGONOMETRIC SERIES
}

\author{
S. N. Mukhopadhyay
}

The symmetric $P^{2 m}$-integral (and $P^{2 m+1}$-integral) as defined by R. D. James in “Generalized $n$th primitives", Trans. Amer. Math. Soc., 76 (1954), is useful to solve problems relating to trigonometric series (see R. D. James: Summable trigonometric series, Pacific J. Math., 6 (1956)). But the definition of the integral is not valid, since Lemma 5.1 of the former paper of James, which is the basis of the whole theory, is incomplete due to the fact that the difference of two functions having property $B_{2 m-2}$ may not have this property. Therefore, all the subsequent results of James also remain incomplete and a complete systematic definition of the integral is needed.

In the present paper a definition of the $P^{2 m}$-integral (and $P^{2 m+1}$-integral) is given and it is shown that all the results of the later paper of James remain valid with this integral.

1. Definitions and Notations. Most of the definitions and notations of [8] will be used with essential modifications. The generalized symmetric derivative [8] (also called symmetric de La Vallée Poussin derivative [18]) of even and odd orders and the generalized unsymmetric derivative [8] (also called Peano derivative [13] or unsymmetric de La Vallée Poussin derivative [11]) of a function $f$ at $x_{0}$ will be denoted by $D^{r} f\left(x_{0}\right)$ and $f_{(r)}\left(x_{0}\right)$ respectively, where $r$ denotes the order of the respective derivatives. If $D^{2 k} f\left(x_{0}\right)$ exists, $0 \leqq k \leqq$ $m-1$, define $\theta_{2 m}\left(f ; x_{0}, h\right)$ by

$$
\frac{h^{2 m}}{(2 m) !} \theta_{2 m}\left(f ; x_{0}, h\right)=\frac{1}{2}\left\{f\left(x_{0}+h\right)+f\left(x_{0}-h\right\}-\sum_{k=0}^{m-1} \frac{h^{2 k}}{(2 k) !} D^{2 k} f\left(x_{0}\right)\right. \text {. }
$$

The upper generalized symmetric derivate of $f$ at $x_{0}$ of order $2 m$ is defined as

$$
\bar{D}^{2 m} f\left(x_{0}\right)=\limsup _{h \rightarrow 0} \theta_{2 m}\left(f ; x_{0}, h\right) .
$$

Replacing 'lim sup' by 'lim inf' one gets the definition of $\underline{D}^{2 m} f\left(x_{0}\right)$.

The function $f$ is said to satisfy the property $\overline{\mathscr{S}}_{2 m}$ at $x_{0}$, written as $f \in \overline{\mathscr{S}}_{2 m}\left(x_{0}\right)$, if

$$
\limsup _{h \rightarrow 0} h \theta_{2 m}\left(f ; x_{0}, h\right) \geqq 0,
$$

and $f \in \mathscr{S}_{2 m}\left(x_{0}\right)$ if $-f \in \overline{\mathscr{S}}_{2 m}\left(x_{0}\right)$. The function $f$ is said to be smooth 
at $x_{0}$ of order $2 m$ if

$$
\lim _{h \rightarrow 0} h \theta_{2 m}\left(f ; x_{0}, h\right)=0 .
$$

Clearly smoothness of order $2 m$ implies smoothness of order $2 m-2$ and if $f$ is smooth at $x_{0}$ of order $2 m$ then $f \in \overline{\mathscr{S}}_{2 m}\left(x_{0}\right) \cap \underline{\mathscr{S}}_{2 m}\left(x_{0}\right)$. For symmetric derivatives of odd order, $\theta_{2 m+1}\left(f ; x_{0}, h\right), \bar{D}^{2 m+1} f\left(x_{0}\right), \underline{D}^{2 m+1} f\left(x_{0}\right)$, $\overline{\mathscr{S}}_{2 m+1}\left(x_{0}\right), \mathscr{S}_{2 m+1}\left(x_{0}\right)$ are defined analogously.

If $f_{(r)}\left(x_{0}\right)$ exists, $0 \leqq r \leqq n-1, \gamma_{n}\left(f ; x_{0}, h\right)$ is defined as

$$
\frac{h^{n}}{n !} \gamma_{n}\left(f ; x_{0}, h\right)=f\left(x_{0}+h\right)-\sum_{r=0}^{n-1} \frac{h^{\gamma}}{r !} f_{(r)}\left(x_{0}\right) .
$$

The upper generalized unsymmetric derivate of $f$ at $x_{0}$ of order $n$ is defined as

$$
\bar{f}_{(n)}\left(x_{0}\right)=\lim _{h \rightarrow 0} \sup \gamma_{n}\left(f ; x_{0}, h\right)
$$

with a similar definition for $f_{(n)}\left(x_{0}\right)$. By restricting $h$ suitably one can define one-sided derivates which are denoted by $\bar{f}_{(n)}^{+}\left(x_{0}\right)$, etc. For convenience, the first order derivates $\bar{f}_{(1)}\left(x_{0}\right), \bar{f}_{(1)}^{+}\left(x_{0}\right)$, etc., will be denoted simply by $\bar{f}\left(x_{0}\right), \bar{f}^{+}\left(x_{0}\right)$, etc. The ordinary $n$th derivative of $f$ at $x_{0}$ will be denoted by $f^{(n)}\left(x_{0}\right)$.

A function $f$ is said to satisfy the property $\mathscr{R}$ in an interval $I$, written $f \in \mathscr{R}$ in $I$, if for every perfect set $P \subset I$, there is a portion of $P$ in which $f$ restricted to $P$ is continuous (see [17]). A function $f$ is said to satisfy the property $\mathscr{T}$ in $(a, b)$, written $f \in \mathscr{T}$ in $(a, b)$, if there exists a function $F$ continuous in $[a, b]$ such that $F_{(n)}=f$ in $(a, b)$ for some $n$. The class of all Darboux functions will be denoted by $\mathscr{D}$. From the properties of Darboux functions it follows that if $D^{2 k} f \in \mathscr{D}$ and if $g$ is continuous then $D^{2 k} f+g \in \mathscr{D}$. This fact will be used in the sequel. For the definition of $n$-convex functions we refer to $[8,1]$.

We now come to the definition of major and minor functions. Let $f$ be defined in $(a, b)$ and let $a=a_{1}<a_{2}<\cdots<a_{2 m}=b$. A function $Q$ is said to be a $P^{2 m}$-major function or simply a major function of $f$ over $\left(a_{i} ; 1 \leqq i \leqq 2 m\right)$ if

(i) $Q$ is continuous in $[a, b]$,

(ii) $D^{2 m-2} Q$ exists and $D^{2 k} \in \mathscr{R} \cap \mathscr{T}$ in $(a, b), 0 \leqq k \leqq m-1$,

(iii) $Q\left(a_{i}\right)=0,1 \leqq i \leqq 2 m$,

(iv) $\underline{D}^{2 m} Q \geqq f$ a.e. in $(a, b)$,

(v) $\underline{D}^{2 m} Q>-\infty$, except on an enumerable set $E \subset(a, b)$,

(vi) $Q$ is smooth of order $2 m$ on $E$.

The function $q$ is a minor function of $f$ if $-q$ is a major function of $-f$. The $P^{2 m+1}$-major functions and $P^{2 m+1}$-minor functions are defined 
similarly.

This definition of major and minor functions differs from that of James [8] in allowing certain exceptional sets in (iv) and (v). But this is standard and is also noted by James in his modified definition of the $P^{2 m}$-integral [9]. Another difference is in condition (ii) where we are assuming $D^{2 k} Q \in \mathscr{R} \cap \mathscr{T}$ instead of James' [8] requirement that $Q$ has properties $A_{2 m}$ and $B_{2 m-2}$. (The property $\mathscr{R}$ is weaker than $A_{2 m}$ by Lemma 3.2 of [8] and the property $\mathscr{T}$ is stronger than $B_{2 m-2}$ by Lemma 8.1 of [8] or by Theorem 2 of [13].) But this is necessary since the difference of two functions in $\mathscr{R} \cap \mathscr{T}$ is in $\mathscr{R} \cap \mathscr{T}$ which is not true with the property $B_{2 m-2}$. We shall prove in the sequel that this is a proper definition of major and minor functions and the $P^{2 m}$-integral defined by these major and minor functions is capable of handling trigonometric series.

\section{Preliminary lemmas.}

LEMMA 2.1. If $f$ is smooth of order $2 m+1$, as well as of order $2 m+2$, at $x_{0}$ then $f_{(2 m)}\left(x_{0}\right)$ exists. If $f_{(n)}\left(x_{0}\right)$ exists then $f$ is smooth of order $n+1$. More generally, if $\underline{f}_{(n+1)}^{+}\left(x_{0}\right), \underline{f}_{(n+1)}\left(x_{0}\right), \bar{f}_{(n+1)}^{+}\left(x_{0}\right), \bar{f}_{(\overline{l n}+1)}\left(x_{0}\right)$ are all finite, then

$$
\begin{aligned}
& \limsup _{h \rightarrow 0} h \theta_{n+2}\left(f ; x_{0}, h\right) \leqq \frac{n+2}{2}\left\{\bar{f}_{(n+1)}^{+}\left(x_{0}\right)-\underline{f}_{(n+1)}\left(x_{0}\right)\right\} \\
& \liminf _{h \rightarrow 0} h \theta_{n+2}\left(f ; x_{0}, h\right) \geqq \frac{n+2}{2}\left\{\underline{f}_{(n+1)}^{+}\left(x_{0}\right)-\bar{f}_{(n+1)}\left(x_{0}\right)\right\} .
\end{aligned}
$$

Proof. The first part is clear. For the last part, since $f_{(n)}\left(x_{0}\right)$ exists, $D^{r} f\left(x_{0}\right)$ exists, $0 \leqq r \leqq n$, and

$$
\begin{aligned}
& \frac{1}{2}\left\{\gamma_{n+1}\left(f ; x_{0}, h\right)+\gamma_{n+1}\left(f ; x_{0},-h\right)\right\}=\theta_{n+1}\left(f ; x_{0}, h\right) \\
& \frac{1}{2}\left\{\gamma_{n+1}\left(f ; x_{0}, h\right)-\gamma_{n+1}\left(f ; x_{0},-h\right)\right\}=\frac{h}{n+2} \theta_{n+2}\left(f ; x_{0}, h\right) .
\end{aligned}
$$

From (2.1)

$$
\lim _{h \rightarrow 0} h \theta_{n+1}\left(f ; x_{0}, h\right)=0,
$$

and from (2.2)

$$
\frac{n+2}{2}\left\{\underline{f}_{(n+1)}^{+}\left(x_{0}\right)-\bar{f}_{\langle(n+1)}\left(x_{0}\right)\right\} \leqq \liminf _{h \rightarrow 0} h \theta_{n+2}\left(f ; x_{0}, h\right) .
$$

The other relation follows similarly. 
LEMMA 2.2. If $G_{(n-1)}\left(x_{0}\right)$ and $D^{n} G\left(x_{0}\right)$ exist and if $G \in \overline{\mathscr{S}}_{n+2}\left(x_{0}\right)$ then the function $\omega_{n+1}\left(G ; x_{0}, h\right)$ defined by

$$
\frac{h^{n+1}}{(n+1) !} \omega_{n+1}\left(G ; x_{0}, h\right)=G\left(x_{0}+h\right)-\sum_{r=0}^{n-1} \frac{h^{r}}{r !} G_{(r)}\left(x_{0}\right)-\frac{h^{n}}{n !} D^{n} G\left(x_{0}\right)
$$

satisfies the relation

$$
\limsup _{h \rightarrow 0+} \omega_{n+1}\left(G ; x_{0}, h\right) \geqq \liminf _{h \rightarrow 0-} \omega_{n+1}\left(G ; x_{0}, h\right) .
$$

Proof. Since

$$
\omega_{n+1}\left(G ; x_{0}, h\right)-\omega_{n+1}\left(G ; x_{0},-h\right)=\frac{2 h}{n+2} \theta_{n+2}\left(G ; x_{0}, h\right),
$$

and since $G \in \overline{\mathscr{S}}_{n+2}\left(x_{0}\right)$, the proof is immediate.

Lemma 2.3. If $f_{(n)}$ exists in $(a, b)$ and $x_{0} \in(a, b)$ then

$$
\begin{gathered}
{\underline{\left(f_{(n)}\right)}}^{+}\left(x_{0}\right) \leqq \underline{f}_{(n+1)}^{+}\left(x_{0}\right), \bar{f}_{(n+1)}^{+}\left(x_{0}\right) \leqq \overline{\left(f_{(n)}\right)^{+}}\left(x_{0}\right), \quad \text { etc. } \\
\underline{\left(f_{(n)}\right)}\left(x_{0}\right) \leqq \underline{D}^{n+1} f\left(x_{0}\right), \bar{D}^{n+1} f\left(x_{0}\right) \leqq \overline{\left(f_{(n)}\right)}\left(x_{0}\right) .
\end{gathered}
$$

Proof. If $n=0$ this is immediate. Suppose $n \geqq 1$. Then $f$ is continuous in $(a, b)$. Let $x_{0} \in[\alpha, \beta] \subset(a, b)$. Then each $f_{(k)}$ is $C_{k^{-}}$ continuous in $[\alpha, \beta], 0 \leqq k \leqq n$, by Lemma 11.1 of [8]. From the definition of Cesaro derivative (see [4]) we have $C_{n} D^{+} f_{(n)}\left(x_{0}\right)=\bar{f}_{(n+1)}^{+}\left(x_{0}\right)$, where $C_{n} D^{+} f_{(n)}\left(x_{0}\right)$ is the right hand upper $n$th Cesaro derivate of $f_{(n)}$ at $x_{0}$. Since $C_{0} D^{+} f_{(n)}\left(x_{0}\right)$ is the first order derivate $\overline{\left(f_{(n)}\right)}\left(x_{0}\right),(2.4)$ follows from Theorem 2.1 of [4]. Lastly, from (2.1), $\underline{D}^{n+1} f\left(x_{0}\right) \geqq$ $f_{(n+1)}\left(x_{0}\right)$ and hence (2.5) follows from (2.4).

Lemma 2.4. Let $g$ be continuous in $[a, b]$ and $\bar{D}^{2} g \geqq 0$ in $(a, b)$, except on an enumerable set $E \subset(a, b)$ and let $g \in \overline{\mathscr{S}}_{2}(x)$ for $x \in E$. Then $g$ is convex in $[a, b]$.

This is proved in [19, I, p. 328], which sharpens a result of de La Vallée Poussin (see [16, Lemma 3]).

3. $2 m$-convex functions. In this section and in $\S 4$, the results are stated in a more general form than is necessary for $P^{2 m}$-major and $P^{2 m+1}$-major functions. Since every member in $\mathscr{T}$ possesses Darboux property [13], we have $\mathscr{T} \cap \mathscr{R} \subset \mathscr{D} \cap \mathscr{R}$ and hence these results are applicable in $\S \S 5$ and 6 .

THeOREm 3.1, 2m. Suppose that 
(i) $f$ is continuous in $[a, b]$,

(ii) $D^{2 m-2} f$ exists and $D^{2 k} f \in \mathscr{D} \cap \mathscr{R}$ in $(a, b), 0 \leqq k \leqq m-1$,

(iii) $\bar{D}^{2 m} f \geqq 0$ in $(a, b)$, except on an enumerable set $E \subset(a, b)$,

(iv) $f \in \overline{\mathscr{S}}_{2 m}(x)$ for $x \in E$.

Then $D^{2 m-2} f$ is convex in $(a, b)$ and it is the continuous derivative $f^{(2 m-2)}$ in $(a, b)$.

The above theorem is true for $m=1$ by Lemma 2.4. So, we assume that the theorem is true for $m=m_{0}$ i.e., Theorem $3.1,2 \mathrm{~m}_{0}$ is true and we prove that Theorem 3.1, $2\left(m_{0}+1\right)$ is also true and so the theorem will be proved to be true for all $m$ by induction on $m$. We require the following auxiliary lemmas:

Lemma $3.1,2 \mathrm{~m}_{0}$. Suppose that

(i) $G$ is continuous in $[a, b]$,

(ii) $D^{2 m_{0}} G$ exists in $(a, b)$ and is $\mathscr{L}$-integrable in $[a, b]$,

(iii) $D^{2 k} G \in \mathscr{D} \cap \mathscr{R}$ in $(a, b), 0 \leqq k \leqq m_{0}-1$.

Then $\Psi-G$ is a polynomial of degree at most $2 m_{0}-1$ in $[a, b]$, where

$$
\begin{aligned}
& \Psi(x)=\frac{1}{\left(2 m_{0}-2\right) !} \int_{a}^{x}(x-t)^{2 m_{0}-2} g(t) d t \\
& g(x)=\int_{a}^{x} D^{2 m_{0}} G(t) d t
\end{aligned}
$$

and $G^{\left(2 m_{0}-1\right)}$ exists and is continuous in $(a, b)$.

Proof. As in [10, Theorem 18], one can construct a sequence of continuous functions $\left\{A_{i}\right\}$ which converges uniformly to $g$ in $[a, b]$ as $i \rightarrow \infty$ and for all $i$

$$
\underline{\left(A_{i}\right)}(x)>D^{2 m_{0}} G(x), \quad x \in(a, b) .
$$

For each $i$, define

$$
U_{i}(x)=\frac{1}{\left(2 m_{0}-2\right) !} \int_{a}^{x}(x-t)^{2 m_{0}-2} A_{i}(t) d t, \quad x \in[a, b] .
$$

Then $\left\{U_{i}\right\}$ converges uniformly to $\Psi$ in $[a, b]$ as $i \rightarrow \infty$. Since $A_{i}$ is continuous, taking $\left(2 m_{0}-1\right)$ th derivative

$$
U_{i}^{\left(2 m_{0}-1\right)}(x)=A_{i}(x), \quad x \in(a, b) .
$$

So, by (2.5) we have

$$
\underline{\left(A_{i}\right)}(x)=\underline{\left(U_{i}^{\left(2 m_{0}-1\right)}\right)}(x) \leqq \underline{D}^{2 m_{0}} U_{i}(x), \quad x \in(a, b) .
$$

Since by construction $\underline{\left(A_{i}\right)}(x)>D^{2 m_{0}} G(x)$ for $x \in(a, b)$, 


$$
\bar{D}^{2 m_{0}}\left[U_{i}-G\right](x)>\bar{D}^{2 m_{0}} U_{i}(x)-\underline{\left(A_{i}\right)}(x), \quad x \in(a, b) .
$$

Hence from (3.1) and (3.2)

$$
\bar{D}^{2 m_{0}}\left[U_{i}-G\right](x)>0, \quad x \in(a, b) .
$$

Since $D^{2 k} G \in \mathscr{D} \cap \mathscr{R}$ and $D^{2 k} U_{i}$ is continuous in $(a, b)$ for $0 \leqq k \leqq$ $m_{0}-1, D^{2 k}\left[U_{i}-G\right] \in \mathscr{D} \cap \mathscr{R}$ in $(a, b)$ for $0 \leqq k \leqq m_{0}-1$. Hence by Theorem 3.1, $2 \mathrm{~m}_{0}, D^{2 m_{0}-2}\left[U_{i}-G\right]$ is convex in $(a, b)$ and so $U_{i}-G$ is $2 m_{0}$-convex in $(a, b)$ and by the continuity, $U_{i}-G$ is $2 m_{0}$-convex in $[a, b]$. Since $U_{i}-G$ converges uniformly to $\Psi-G$ in $[a, b], \Psi-G$ is $2 m_{0}$-convex in $[a, b]$. It can be similarly shown that $\Psi-G$ is $2 m_{0^{-}}$ concave in $[a, b]$. Hence $\Psi-G$ is a polynomial of degree at most $2 m_{0}-1$. Since $\Psi^{\left(2 m_{0}-1\right)}$ exists and is continuous, $G^{\left(2 m_{0}-1\right)}$ also exists and is continuous in $(a, b)$.

LemMa $3.2,2 \mathrm{~m}_{0}$. Let $G$ be continuous in $[a, b]$ and let $D^{2 m_{0}} G$ exist in $(a, b)$ and be $\mathscr{L}$-integrable in $[a, b]$. Let $G^{\left(2 m_{0}-1\right)}$ exist and be continuous in $(a, b)$. If $D^{2 m_{0}} G$ attains a maximum at $x_{0} \in(a, b)$ then

$$
\limsup _{h \rightarrow 0+} \omega_{2 m_{0}+1}\left(G ; x_{0}, h\right) \leqq 0 \leqq \liminf _{h \rightarrow 0-} \dot{\omega}_{2 m_{0}+1}\left(G ; x_{0}, h\right),
$$

where $\omega$ is the function defined in (2.3) with $n=2 m_{0}$.

Proof. Let

$$
J(x)=\int_{a}^{x} D^{2 m_{0}} G(t) d t, \quad x \in[a, b] .
$$

Then by Lemma $3.1,2 \mathrm{~m}_{0} J-G^{\left(2 m_{0}-1\right)}$ is constant. Since $G^{\left(2 m_{0}-1\right)}$ is continuous in $(a, b)$, by mean value property, for any $h, 0<h<b-x_{0}$, there is $\eta, 0<\eta<1$, such that

$$
\begin{aligned}
\omega_{2 m_{0}+1}\left(G ; x_{0}, h\right) & =\frac{2}{(\eta h)^{2}}\left\{G^{\left(2 m_{0}-1\right)}\left(x_{0}+\eta h\right)-G^{\left(2 m_{0}-1\right)}\left(x_{0}\right)-\eta h D^{2 m_{0}} G\left(x_{0}\right)\right\} \\
& =\frac{2}{(\eta h)^{2}} \int_{x_{0}}^{x_{0}+\eta h}\left\{D^{2 m_{0}} G(t)-D^{2 m_{0}} G\left(x_{0}\right)\right\} d t .
\end{aligned}
$$

Therefore, since $D^{2 m_{0}} G$ is maximum at $x_{0}$,

$$
\limsup _{h \rightarrow 0+} \omega_{2 m_{0}+1}\left(G ; x_{0}, h\right) \leqq 0 \text {. }
$$

The other part follows similarly.

Lemma $3.3,2 \mathrm{~m}_{0}$. Suppose that

(i) $F$ is continuous in $[a, b]$, 
(ii) $D^{2 m_{0}-2} F$ exists and $D^{2 k} F \in \mathscr{D} \cap \mathscr{R}$ in $(a, b), 0 \leqq k \leqq m_{0}-1$,

(iii) $\bar{D}^{2 m_{0}} F \geqq 0$ in $(a, b)$, except on an enumerable set $E \subset(a, b)$,

(iv) $F \in \overline{\mathscr{S}}_{2 m_{0}}(x)$ for $x \in E$.

Then

$$
\theta_{2 m_{0}}(F ; x, h) \geqq 0, \text { for all } x, h, a<x-h<x+h<b .
$$

LEMMA $3.4,2 \mathrm{~m}_{0}$. Suppose that

(i) $G$ is continuous in $[a, b]$,

(ii) $D^{2 m_{0}} G$ exists and $D^{2 k} G \in \mathscr{D} \cap \mathscr{R}$ in $(a, b), 0 \leqq k \leqq m_{0}$,

(iii) $D^{2 m_{0}} G$ attains a maximum at $x_{0} \in(a, b)$. Then

$$
\bar{D}^{2 m_{0}+2} G\left(x_{0}\right) \leqq 0
$$

The proof of Lemma $3.3,2 \mathrm{~m}_{0}$ is similar to that of Lemma $4.1,2 \mathrm{~m}_{0}$ of [8]. Lemma $3.4,2 \mathrm{~m}_{0}$ can be proved by using Lemma $3.3,2 \mathrm{~m}_{0}$ in the same manner as in Lemma $4.2,2 \mathrm{~m}_{0}$ of [8].

Lemma $3.5,2 \mathrm{~m}_{0}$. Suppose that

(i) $f$ is continuous in $[a, b]$,

(ii) $D^{2 m_{0}} f$ exists and $D^{2 k} f \in \mathscr{D} \cap \mathscr{R}$ in $(a, b), 0 \leqq k \leqq m_{0}$,

(iii) $\bar{D}^{2 m_{0}+2} f \geqq 0$ in $(a, b)$, except on an enumerable set $E \subset(a, b)$,

(iv) $f \in \overline{\mathscr{S}}_{2 m_{0}+2}(x)$ for $x \in E$,

(v) $D^{2 m_{0}} f$ is upper semicontinuous in $(a, b)$ and $\mathscr{L}$-integrable in $[a, b]$.

Then $D^{2 m_{0}} f$ is convex in $(a, b)$.

Proof. We first consider the special case when the inequality in (iii) is strict inequality. Suppose that $D^{2 m_{0}} f$ is not convex in $(a, b)$. Then there is a subinterval $[\alpha, \beta] \subset(a, b)$ such that

$$
\begin{aligned}
\rho(x) & =D^{2 m_{0}} f(x)-\frac{1}{\beta-\alpha}\left\{(\beta-x) D^{2 m_{0}} f(\alpha)+(x-\alpha) D^{2 m_{0}} f(\beta)\right\} \\
& =D^{2 m_{0}} f(x)-p x-q
\end{aligned}
$$

takes positive values somewhere in $(\alpha, \beta)$. Since $\rho$ is upper semicontinuous in $[\alpha, \beta]$ and $\rho(\alpha)=\rho(\beta)=0, \rho$ attains maximum in $(\alpha, \beta)$. So, if $\mu$ is sufficiently near to $p$ then the function $D^{2 m_{0}} G$, where

$$
G(x)=f(x)-\mu \frac{x^{2 m_{0}+1}}{\left(2 m_{0}+1\right) !}-q \frac{x^{2 m_{0}}}{\left(2 m_{0}\right) !},
$$

also attains its maximum in $(\alpha, \beta)$, say, at $x_{\mu}$. Hence by Lemma $3.4,2 \mathrm{~m}_{0}$ 


$$
\bar{D}^{2 m_{0}+2} G\left(x_{\mu}\right)=\bar{D}^{2 m_{0}+2} f\left(x_{\mu}\right) \leqq 0 .
$$

Hence $x_{\mu} \in E$. Now $G$ satisfies the hypotheses of Lemma 3.1, $2 \mathrm{~m}_{0}$ and hence $G^{\left(2 m_{0}-1\right)}$ exists and is continuous in $(a, b)$. Also since $f \in \overline{\mathscr{S}}_{2 m_{0}+2}(x)$ for $x \in E, G \in \overline{\mathscr{S}}_{2 m_{0}+2}\left(x_{\mu}\right)$. Hence by Lemma 2.2

$$
\limsup _{h \rightarrow 0+} \omega_{2 m_{0}+1}\left(G ; x_{\mu}, h\right) \geqq \liminf _{h \rightarrow 0-} \omega_{2 m_{0}+1}\left(G ; x_{\mu}, h\right)
$$

where $\omega$ is the function as defined in (2.3) with $n=2 m_{0}, x_{0}=x_{\mu}$. But by Lemma $3.2,2 \mathrm{~m}_{0}$, since $D^{2 m_{0}} G$ is maximum at $x_{\mu}$,

$$
\operatorname{lim\operatorname {sup}} \omega_{2 m_{0}+1}\left(G ; x_{\mu}, h\right) \leqq 0 \leqq \liminf _{h \rightarrow 0-} \omega_{2 m_{0}+1}\left(G ; x_{\mu}, h\right)
$$

and hence

$$
\liminf _{h \rightarrow 0-} \omega_{2 m_{0}+1}\left(G ; x_{\mu}, h\right)=0
$$

i.e.,

$$
\liminf _{h \rightarrow 0-} \omega_{2 m_{0}+1}\left(f ; x_{\mu}, h\right)=\mu .
$$

Thus for each $\mu$ sufficiently near to $p$ there exists $x_{\mu} \in E$ and for different $\mu$ the points $x_{\mu}$ are also different. This contradicts the fact that $E$ is enumerable.

To complete the proof, consider, for arbitrary $\varepsilon>0$, the function $g_{\varepsilon}$ where

$$
g_{\varepsilon}(x)=f(x)+\varepsilon \cdot \frac{x^{2 m_{0}+2}}{\left(2 m_{0}+2\right) !} .
$$

Then by the above special case, $D^{2 m_{0}} g_{\varepsilon}$ is convex in $(a, b)$ and since $\varepsilon$ is arbitrary, $D^{2 m_{0}} f$ is convex in $(a, b)$, completing the proof.

Proof of Theorem 3.1, $2\left(m_{0}+1\right)$. To prove the theorem we remark that under the hypotheses, if $D^{2 m_{0}} f$ is continuous in an interval $(\alpha, \beta) \subset$ $(a, b)$, then by Lemma 3.1, $2 \mathrm{~m}_{0}, f^{\left(2 m_{0}-1\right)}$ exists and is continuous in $(\alpha, \beta)$ and so by Lemma 7 of [18], $D^{2 m_{0}} f$ is the continuous ordinary derivative $f^{\left(2 m_{0}\right)}$ in $(\alpha, \beta)$. Hence applying the mean value property it can be shown that $\bar{D}^{2}\left(f^{\left(2 m_{0}\right)}\right) \geqq \bar{D}^{2 m_{0}+2} f$ and that $f^{\left(2 m_{0}\right)} \in \overline{\mathscr{S}}_{2}(x)$ if $f \in \overline{\mathscr{S}}_{2 m_{0}+2}(x)$ for points in $(\alpha, \beta)$ and so by Lemma $2.4, f^{\left(2 m_{0}\right)}$ is convex in $(\alpha, \beta)$.

Let $U$ be the set of all points $x$ in $(a, b)$ such that there is a neighborhood of $x$ in which $D^{2 m_{0}} f$ is continuous. Then $U$ is open. Let $(\alpha, \beta)$ be any component interval of $U$. Then $D^{2 m_{0}} f$ is continuous in $(\alpha, \beta)$ and so by the above remark $D^{2 m_{0}} f$ is convex in $(\alpha, \beta)$. Hence $\lim _{x \rightarrow \alpha_{+}} D^{2 m_{0}} f(x)$ and $\lim _{x \rightarrow \beta-} D^{2 m_{0}} f(x)$ exist and by the property $\mathscr{D}$, $D^{2 m_{0}} f$ is continuous in $[\alpha, \beta] \cap(a, b)$. Let $P=(a, b)-U$. Then $P$ is 
closed in $(a, b)$. Since $D^{2 m_{0}} f$ is continuous in the closure (relative to $(a, b))$ of each component interval of $U, P$ is perfect in $(a, b)$. If possible, suppose that $P \neq 0$. Then there is $[c, d] \subset(a, b)$ such that $[c, d] \cap P$ is a nonvoid perfect set. Since $D^{2 k} f \in \mathscr{R}$ in $(a, b)$, there is a portion of $[c, d] \cap P$, say, $H=\left[a_{0}, b_{0}\right] \cap P$ on which $D^{2 k} f / H$ is continuous for each $k, 0 \leqq k \leqq m_{0}$. It can be shown, as in Theorem 4.1, $2\left(m_{0}+1\right)$ of [8] that $D^{2 m_{0}} f$ is upper semicontinuous in $\left[a_{0}, b_{0}\right]$. Hence there is $M$ such that $D^{2 m_{0}} f(x) \leqq M$ for $x \in\left[a_{0}, b_{0}\right]$. Since the theorem is true for $m=m_{0}$, the function $F(x)=M x^{2} / 2-D^{2 m_{0}-2} f(x)$ is convex in $\left(a_{0}, b_{0}\right)$. Choose $a_{1}, b_{1}$, such that $a_{0}<a_{1}<b_{1}<b_{0}$ and $P \cap\left(a_{1}, b_{1}\right) \neq 0$. Then by Lemma 3.16 of [19, I, p. 328], $D^{2} F$ exists almost everywhere in $\left(a_{0}, b_{0}\right)$ and is $\mathscr{L}$-integrable in $\left[a_{1}, b_{1}\right]$. Since $F$ is continuous, $D^{2} F=M-D^{2 m_{0}} f$ holds whenever $D^{2} F$ exists and hence $D^{2 m_{0}} f$ is $\mathscr{L}$-integrable in $\left[a_{1}, b_{1}\right]$. So, by Lemma $3.5,2 \mathrm{~m}_{0}, D^{2 m_{0}} f$ is convex in $\left(a_{1}, b_{1}\right)$. Hence $D^{2 m_{0}} f$ is continuous in $\left(a_{1}, b_{1}\right)$. This contradicts the fact that $\left(a_{1}, b_{1}\right) \cap P \neq 0$. Hence $P=0$ and so $D^{2 m_{0}} f$ is continuous in $(a, b)$. Hence by our earlier remark $D^{2 m_{0}} f$ is convex in $(a, b)$. The rest follows from Lemma $3.1,2 \mathrm{~m}_{0}$ and Lemma 7 of [18]. This completes the proof of the theorem for $m=m_{0}+1$.

Thus the theorem is true for all $m$ and so henceforth we shall omit $2 m$ in refering to this theorem. The usual extension of the above theorem is the following

THEOREM 3.2. Suppose that

(i) $f$ is continuous in $[a, b]$,

(ii) $D^{2 m-2} f$ exists and $D^{2 k} f \in \mathscr{D} \cap \mathscr{R}$ in $(a, b), 0 \leqq k \leqq m-1$,

(iii) $\bar{D}^{2 m} f \geqq 0$ a.e. in $(a, b)$,

(iv) $\bar{D}^{2 m} f>-\infty$, except on an enumerable set $E \subset(a, b)$,

(v) $f \in \overline{\mathscr{S}}_{2 m}(x)$, for $x \in E$.

Then $D^{2 m-2} f$ is convex in $(a, b)$ and $D^{2 m-2} f$ is the continuous derivative $f^{(2 m-2)}$ in $(a, b)$.

This can be proved from Theorem 3.1 by using standard argument used to prove Theorem 1.1 of [5] or Theorem 16 of [1] and so we omit it.

REMARK 3.1. The property $D^{2 k} f \in \mathscr{D}$ for $0 \leqq k \leqq m-1$, in the above theorem plays an important role. For, consider the function $f$ where

$$
f(x)= \begin{cases}x^{2}, & x \geqq 0 \\ -x^{2}, & x<0 .\end{cases}
$$

Then $D^{2} f$ exists everywhere but $D^{2} f \notin \mathscr{D}$. Also $f$ satisfies all the other conditions of the above theorem and $D^{4} f=0$ everywhere; but $D^{2} f$ is 
neither convex nor concave in any interval including 0 .

REMARK 3.2. The above example shows that if $\underline{D}^{2 m} f$ replaces $\bar{D}^{2 m} f$ in the hypotheses (iii) and (iv) of the above theorem and if in (v) smoothness of $f$ of order $2 m$ is assumed everywhere, then even under this stronger conditions the theorem is false without the property $D^{2 k} f \in \mathscr{D}$.

4. $(2 m+1)$-convex functions. Now it is natural to ask whether the analogous results hold for odd order derivatives. In [8], it is indicated that the proof of Theorem 4.1, 3 of [8] was similar to that of a theorem of Saks [14]. But Saks used the lower derivate $\underline{D}^{3} f$ and not $\bar{D}^{3} f$ and so the induction on $m$ in [8] ensures the validity of Theorem $4.1,2 m+1$ of [8], provided $\bar{D}^{2 m+1} f$ is replaced by $\underline{D}^{2 m+1} f$ in its hypotheses. But if in the hypotheses of Theorem 4.1,2m +1 of [8], $\bar{D}^{2 m+1} f$ is replaced by $\underline{D}^{2 m+1} f$ then this new theorem is only a consequence of Theorem 4.1, $2(m+1)$ of [8] for the integrated function. The proof of Theorem 4.1, $2 m+1$ of [8] is thus incomplete. We complete the proof in the following more general theorem.

Theorem 4.1. Suppose that

(i) $f$ is continuous in $[a, b]$,

(ii) $D^{2 m-1} f$ exists and $D^{2 k+1} f \in \mathscr{D} \cap \mathscr{R}$ in $(a, b), 0 \leqq k \leqq m-1$,

(iii) $\bar{D}^{2 m+1} f \geqq 0$ in $(a, b)$, except on an enumerable set $E \subset(a, b)$,

(iv) $f \in \overline{\mathscr{S}}_{2 m+1}(x)$ for $x \in E$.

Then $D^{2 m-1} f$ is convex in $(a, b)$ and it is the continuous derivative $f^{(2 m-1)}$ in $(a, b)$.

The proof is similar to that of Theorem 3.1. It is necessary to prove this theorem for $m=1$ and to do this, Lemmas $4.1,1,4.2,1$, $4.4,1,4.5,1$, which are analogous to Lemmas $3.1,2 \mathrm{~m}_{0}, 3.2,2 \mathrm{~m}_{0}, 3.4,2 \mathrm{~m}_{0}$, $3.5,2 \mathrm{~m}_{0}$, will be needed. The proofs of Lemmas $4.2,1$ and 4.5, 1 are similar to those of Lemmas $3.2,2 \mathrm{~m}_{0}$ and $3.5,2 \mathrm{~m}_{0}$ respectively. In proving Lemma 4.1, 1 one is to appeal to a result of [12] instead of assuming Theorem $3.1,2 \mathrm{~m}_{0}$ as it was done in Lemma $3.1,2 \mathrm{~m}_{0}$ and in proving Lemma $4.4,1$ one is to notice that since $D^{1} G \in \mathscr{D}$, by the same result of [12], $D^{1} G$ has mean value property and hence for any $h$ there is $\xi, x_{0}-h<\xi<x_{0}+h$, such that

$$
h^{2} \theta_{3}\left(G ; x_{0}, h\right)=3 !\left\{D^{1} G(\xi)-D^{1} G\left(x_{0}\right)\right\} \leqq 0
$$

giving $\bar{D}^{3} G\left(x_{0}\right) \leqq 0$. The proof of Theorem 4.1 for $m=1$ will now follow the same line of argument as in Theorem 3.1, $2\left(m_{0}+1\right)$. The $\mathscr{L}$-integrability of $D^{1} f$ will follow from the fact that $F(x)=M x-$ $f(x)$ is nondecreasing in $\left[a_{0}, b_{0}\right],[12]$ and $M-D^{1} f$ is the derivative of 
$F$ where it exists. Proving the above theorem for $m=1$ and supposing it to be true for $m=m_{0}$, all the lemmas beginning 4.1, $2 m_{0}+1$ through 4.5, $2 m_{0}+1$ can be proved and the proof of the theorem for $m=m_{0}+1$ can be completed. We remark that an analogue of Theorem 3.2 is also true in this case.

5. The $P^{2 m}$-integral. We now come to the definition of the integral. We must show that the definition of major and minor functions, as introduced earlier, actually helps to obtain a proper definition of the integral. For, because of the presence of the exceptional set $E$ in condition (v) and (vi) of the definition of major function we cannot apply directly Theorem 3.2 to prove that $Q-q$ is a $2 m$-convex function for arbitrary major and minor functions $Q$ and $q$ respectively. (As the definition of the $P^{2 m}$-integral in [9] and that of the $P^{2}$-integral in [7] are also affected by the exceptional sets $S$ and $E_{0}$ respectively, (see [9] and [7]) they would also need this clarification; but the definition of the $P^{2}$-integral in [6] is not affected since the smoothness of major and minor functions is assumed everywhere). We shall follow the method adopted in [15].

Lemma 5.1. Given $\varepsilon_{0}>0$ and $x_{0} \in(a, b)$ there is a major function $Q$ for the function $t(x) \equiv 0$ such that

(i) $Q^{(2 m-2)}$ is continuous in $[a, b]$,

(ii) $\underline{D}^{2} Q^{(2 m-2)} \geqq 0$ in $(a, b)$,

(iii) $\lim _{h \rightarrow 0} h \theta_{2}\left(Q^{(2 m-2)} ; x_{0}, h\right)>0, \lim _{h \rightarrow 0} h \theta_{2}\left(Q^{(2 m-2)} ; x, h\right)=0$, for $x \neq x_{0}$,

(iv) $\left|Q^{(2 m-2)}\right| \leqq \varepsilon_{0}$ in $(a, b)$,

(v) $\left|h \theta_{2}\left(Q^{(2 m-2)} ; x, h\right)\right| \leqq \varepsilon_{0}$, for $x \neq x_{0}$, and $x, x \pm h \in(a, b)$.

Proof. Let $g$ be the function such that

$$
\begin{array}{r}
g\left(x_{0}\right)=0, \quad g(a)=\frac{1}{2} \min \cdot\left[\varepsilon_{0}\left(x_{0}-a\right), \frac{\varepsilon_{0}}{(2 m) !}\right], \\
g(b)=\frac{1}{2} \min \cdot\left[\varepsilon_{0}\left(b-x_{0}\right), \frac{\varepsilon_{0}}{(2 m) !}\right],
\end{array}
$$

and $g$ is linear and continuous in each of the interval $\left[a, x_{0}\right]$ and $\left[x_{0}, b\right]$ and let $G$ be the $(2 m-2)$ th indefinite integral of $g$ in $[a, b]$. Then the function $Q$ defined by

$$
Q(x)=G(x)-\sum_{i=1}^{2 m} \lambda\left(x ; a_{i}\right) G\left(a_{\imath}\right)
$$

satisfies the requirements, where

$$
\lambda\left(x ; a_{i}\right)=\prod_{\substack{j=1 \\ j \neq i}}^{2 m} \frac{x-a_{j}}{a_{i}-a_{j}}, \quad a=a_{1}<a_{2}<\cdots<a_{2 m}=b .
$$


LEMMA 5.2. If $Q$ is a major function of $f$ and $\varepsilon>0$, then there is a major function $Q_{\varepsilon}$ such that

$$
\left|D^{2 m-2} Q_{\varepsilon}-D^{2 m-2} Q\right| \leqq \varepsilon, \quad \underline{D}^{2 m} Q_{\varepsilon}>-\infty, \quad \text { in }(a, b) .
$$

Proof. Let $x_{1}, x_{2}, \cdots, x_{k}, \cdots$ be an enumeration of the exceptional set $E \subset(a, b)$, where $\underline{D}^{2 m} Q=-\infty$ holds. For each positive integer $k$, let $F_{k}$ be the major function obtained from Lemma 5.1 with $\varepsilon_{0}$ and $x_{0}$ replaced by $\varepsilon / 2^{k}$ and $x_{k}$ respectively. Set

$$
\Psi(x)=\sum_{k=1}^{\infty} F_{k}^{(2 m-2)}(x), \quad F(x)=\sum_{k=1}^{\infty} F_{k}(x) .
$$

The first series being uniformly and absolutely convergent, $\Psi$ is continuous and $\Psi=F^{(2 m-2)}$. By the mean value property there is $\eta$, $0<\eta<1$, such that

$$
\theta_{2 m}(F ; x, h)=\theta_{2}(\Psi ; x, \eta h)=\sum_{k=1}^{\infty} \theta_{2}\left(F_{k}^{(2 m-2)} ; x, \eta h\right)
$$

and since by (i), (ii) of Lemma 5.1 and by Theorem 3.1, each $F_{k}^{(2 m-2)}$ is convex in $(a, b), D^{2 m} F \geqq 0$ in $(a, b)$. Also, for $x_{i} \in E$, the series $\sum_{k=i+1}^{\infty} h \theta_{2}\left(F_{k}^{(2 m-2)} ; x_{i}, \bar{h}\right)$ is uniformly and absolutely convergent with respect to $h$ and hence

$$
\begin{aligned}
\lim _{h \rightarrow 0} h \theta_{2 m}\left(F ; x_{i}, h\right) & =\lim _{h \rightarrow 0} h \theta_{2}\left(\Psi ; x_{i}, h\right) \\
& =\lim _{h \rightarrow 0} \sum_{k=1}^{\infty} h \theta_{2}\left(F_{k}^{(2 m-2)} ; x_{i}, h\right) \\
& =\lim _{h \rightarrow 0} h \theta_{2}\left(F_{i}^{(2 m-2)} ; x_{i}, h\right) \\
& >0 .
\end{aligned}
$$

Now set

$$
Q_{\varepsilon}(x)=Q(x)+F(x)
$$

Then if $x_{i} \in E$,

$$
\lim _{h \rightarrow 0} h \theta_{2 m}\left(Q_{\varepsilon} ; x_{i}, h\right)=\lim _{h \rightarrow 0} h \theta_{2 m}\left(F ; x_{i}, h\right)>0
$$

and hence $\underline{D}^{2 m} Q_{\varepsilon}\left(x_{i}\right)=\infty$. Clearly $Q_{\varepsilon}$ is a major function of $f$ and by construction $\left|D^{2 m-2} Q_{\varepsilon}-D^{2 m-2} Q\right| \leqq \varepsilon$.

LEMMA 5.3. If $Q$ and $q$ are any major and minor functions then $Q-q$ is $2 m$-convex.

Proof. By Lemma 5.2, for each positive integer $n$ there is a major function $Q_{n}$ and a minor function $q_{n}$ such that 


$$
\left|D^{2 m-2} Q_{n}-D^{2 m-2} Q\right| \leqq \frac{1}{n}, \underline{D}^{2 m} Q_{n}>-\infty, \quad \text { in }(a, b)
$$

and a similar relation for $q_{n}$ holds. Hence $\underline{D}^{2 m}\left[Q_{n}-q_{n}\right] \geqq 0$ a.e. in $(a, b)$ and $\underline{D}^{2 m}\left[Q_{n}-q_{n}\right]>-\infty$ in $(a, b)$. Since $D^{2 k} Q_{n} \in \mathscr{T}$, and $D^{2 k} q_{n} \in$ $\mathscr{T}$, we have $D^{2 k}\left[Q_{n}-q_{n}\right] \in \mathscr{T}$ and hence $D^{2 k}\left[Q_{n}-q_{n}\right] \in \mathscr{D}$, for each $k, 0 \leqq k \leqq m-1,[13]$. So, by Theorem $3.2 D^{2 m-2}\left[Q_{n}-q_{n}\right]$ is convex in $(a, b)$ and hence by $(5.2)$ and a relation for $q_{n}, D^{2 m-2}[Q-q]$ is convex in $(a, b)$ and so the result follows.

Lemma 5.3 gives the analogue of Lemma 5.1 of [8]. Once this lemma is proved all the subsequent results of [8] can be deduced with this definition of major and minor functions. The definition of $P^{2 m}$ integral thus obtained remains valid and all the results of [8] except Theorem 5.4 of [8] are true. We state Theorem 5.4 of [8] in our setting whose proof is similar to that in [8].

THEOREM 5.1. If $G$ is such that

(i) $G$ is continuous in $[a, b]$,

(ii) $D^{2 m-2} G$ exists and $D^{2 k} G \in \mathscr{R} \cap \mathscr{T}$ in $(a, b), 0 \leqq k \leqq m-1$,

(iii) $D^{2 m} G$ exists a.e. in $(a, b)$,

(iv) $-\infty<\underline{D}^{2 m} G \leqq \bar{D}^{2 m} G<\infty$, except on an enumerable set $E \subset$ $(a, b)$,

(v) $G$ is smooth of order $2 m$ on $E$, then $D^{2 m} G$ is $P^{2 m}$-integrable over $\left(a_{i} ; x\right)$, where $a \leqq a_{1}<a_{2}<\cdots<$ $a_{2 m} \leqq b$, and if $a_{r} \leqq x<a_{r+1}$, then

$$
(-1)^{r} \int_{\left(a_{i}\right)}^{x} f(t) d_{2 m} t=G(x)-\sum_{i=1}^{2 m} \lambda\left(x ; a_{i}\right) G\left(a_{i}\right)
$$

where $\lambda$ is the function defined in (5.1).

6. The $P^{2 m+1}$-integral. The definition of $P^{2 m+1}$-integral can be obtained from the $P^{2 m+1}$-major and minor functions in the same manner as in the case of $P^{2 m}$-integral. The $P^{1}$-integral i.e., $P^{2 m+1}$-integral for $m=0$ is not defined in [8]. Theorem 3 of [12] shows that the definition of $P^{1}$-integral is also valid and so the definition of symmetric $P^{n}$-integral is valid for all $n \geqq 1$.

7. The unsymmetric $P^{n}$-integral. The unsymmetric $P^{n}$-integral as defined in [8] is not affected by Lemma 5.1 of [8]. We state here the conditions to be satisfied by an unsymmetric $P^{n}$-major function $Q$ of the function $f$ in our improved setting:

(i) $Q$ is continuous in $[a, b]$,

(ii) $Q_{(n-1)}$ exists in $(a, b)$,

(iii) $Q\left(a_{i}\right)=0,1 \leqq i \leqq n$,

(iv) $\underline{Q}_{(n)} \geqq f$ a.e. in $(a, b)$, 
(v) $\underline{Q}_{(n)}>-\infty$, except on an enumerable set $E \subset(a, b)$. It is easy to verify that for any major and minor function, $Q$ and $q$, the difference $Q-q$ is $n$-convex. The definition of the unsymmetric $P^{n}$-integral now follows that of the symmetric $P^{n}$-integral. For different approach we refer to $[2,3]$.

8. Application to trigonometric series. Now we shall show that the results of [9] remain true with this definition of the $P^{2 m}$-integral. For the notations $A_{n}^{k}(x), B_{n}^{k}(x)$ and the upper and the lower $(C, k)$ sums $S^{k}(x)$ and $s^{k}(x)$, which we shall use here, we refer to [9] (see also [19, I, pp. 74-77]).

Theorem 8.1. (Cf. Theorem 6.2 of [9].) Suppose that the series

$$
\frac{1}{2} a_{0}+\sum_{n=1}^{\infty}\left(a_{n} \cos n x+b_{n} \sin n x\right)
$$

is summable $(C, k)$ almost everywhere to a finite function $f$ on $[0,2 \pi]$ and let

$$
-\infty<s^{k}(x) \leqq S^{k}(x)<\infty,
$$

except on an enumerable set in $[0,2 \pi]$. If for $x \in[0,2 \pi]$

$$
A_{n}^{k-1}(x)=o\left(n^{k}\right), \quad B_{n}^{k-1}(x)=o\left(n^{k}\right),
$$

as $n \rightarrow \infty$, then $f(x), f(x) \cos r x, f(x) \sin r x$, are $P^{k+2}$ integrable over $\left(\alpha_{i} ; x\right)$ and the coefficients of (8.1) are given by

$$
\begin{aligned}
& a_{r}=\frac{\delta_{k}}{2^{k+1} \pi^{k+2}} \int_{\left(\alpha_{i}\right)}^{0} f(x) \cos r x d_{k+2} x \\
& b_{r}=\frac{\delta_{k}}{2^{k+1} \pi^{k+2}} \int_{\left(\alpha_{i}\right)}^{0} f(x) \sin r x d_{k+2} x
\end{aligned}
$$

where

$$
\begin{array}{rlr}
\delta_{k} & =\frac{(k+2) !}{\left\{\left(\frac{k+2}{2}\right) !\right\}^{2}} \quad \text { if } k \text { is even }, \\
& =\frac{(k+2) !}{\left(\frac{k+1}{2}\right) !\left(\frac{k+3}{2}\right) !} \quad \text { if } k \text { is odd. }
\end{array}
$$

Proof. Since (8.1) is summable $(C, k)$, the series obtained by integrating (8.1) term by term $k+2$ times converges uniformly to a continuous function $F$ and

$$
a_{n}=o\left(n^{k}\right), \quad b_{n}=o\left(n^{k}\right),
$$


as $n \rightarrow \infty$, (see [18]) and hence $F$ is smooth of order $k+2$ (see [9, Theorem 3.1]). Since the once-integrated series of (8.1) is also summable $(C, k-1)$ a.e. in $[0,2 \pi]$ (see [11]), $F$ is smooth of order $k+1$; hence by Lemma 2.1, $F_{(k)}$ exists and by Lemma 6 of [18], $F_{(i)} \in \mathscr{R}$ in $(0,2 \pi)$ for $0 \leqq i \leqq k$. By [18, Theorem B] we get from (8.2)

$$
-\infty<\underline{D}^{k+2} F(x) \leqq \bar{D}^{k+2} F(x)<\infty
$$

except on an enumerable set and $D^{k+2} F(x)=f(x)$ a.e. in $(0,2 \pi)$. So, by Theorem 5.1, $f$ is $P^{k+2}$-integrable over $\left(\alpha_{i} ; x\right)$. The proofs that $f(x) \cos r x$ and $f(x) \sin r x$ are also $P^{k+2}$-integrable and that the coefficients of (8.1) are given by (8.4) and (8.5) are similar to those given in [9, Theorem 4.2 and its corollary].

\section{REFERENCES}

1. P.S. Bullen, A criterion for n-convexity, Pacific J. Math., 36 (1971), 81-98.

2. The $P^{n}$-integral, J. Australian Math. Soc., (2), 14 (1972), 219-236.

3. P.S. Bullen and S. N. Mukhopadhyay, Peano derivatives and general integrals, Pacific J. Math., 47 (1973), 43-58.

4. J. C. Burkill, The Cesaro-Perron scale of integration, Proc. London Math. Soc., (2), 39 (1935), 541-552.

5. - Integrals and trigonometric series, Proc. London Math. Soc., (3), 1 (1951), $46-57$.

6. R. D. James and W. H. Gage, A generalized integral, Trans. Royal Soc. Canada, Sec. III, 40 (1946), 25-35.

7. R. D. James, A generalized integral II, Canad. J. Math., 2 (1950), 297-306.

8. —, Generalized $n$th primitives, Trans. Amer. Math. Soc., 76 (1954), 149-176.

9. - Summable trigonometric series, Pacific J. Math., 6 (1956), 99-110.

10. R. L. Jeffery, Trigonometric Series, Toronto, 1956.

11. J. Marcinkeiwicz and A. Zygmund, On the differentiability of functions and summability of trigonometric series, Fundamenta Mathematicae, 26 (1936), 1-43.

12. S. N. Mukhopadhyay, On Schwarz differentiability IV, Acta Mathematica Academiae Scientiarum Hungaricae, 17 (1966), 129-136.

13. H. W. Oliver, The exact Peano derivative, Trans. Amer. Math. Soc., 76 (1954), 444456 .

14. S. Saks, On generalized derivatives, J. London Math. Soc., 7 (1933), 247-251.

15. S. J. Taylor, An integral of Perron's type defined with the help of trigonometric series, Quarterly J. Math., (Oxford), (2), 6 (1955), 255-274.

16. S. Verblunsky, On the theory of trigonometric series II, Proc. London Math. Soc., 34 (1932), 457-491.

17. - On the theory of trigonometric series $V$, Fundamenta Mathematicae, 21 (1933), 168-210.

18. F. Wolf, Summable trigonometric series: an extension of uniqueness theorem, Proc. London Math. Soc., (2), 45 (1939), 328-356.

19. A. Zygmund, Trigonometric series, Cambridge, 1968.

Received January 7, 1974 and in revised form September 25, 1974. The ideas of this work were originated when the author was in a visiting position at The University of British Columbia supported by the National Research Council of Canada Grant No. 672986. The author wishes to thank Professor P. S. Bullen for various helpful discussions.

The University of Burdwan, Burdwan, West Bengal, India 



\section{PACIFIC JOURNAL OF MATHEMATICS}

EDITORS

RICHARD ARENS (Managing Editor)

University of California

Los Angeles, Calıfornia 90024

R. A. Beaumont

University of Washington

Seattle, Washington 98105
J. DugundJI

Department of Mathematics

University of Southern California

Los Angeles, California 90007

D. Gilbarg and J. Milgram

Stanford University

Stanford, California 94305

\section{ASSOCIATE EDITORS}
E. F. BECKENBACH
B. H. NeumanN
F. WOLF
K. YOSHIDA

\section{SUPPORTING INSTITUTIONS}

UNIVERSITY OF BRITISH COLUMBIA

CALIFORNIA INSTITUTE OF TECHNOLOGY

UNIVERSITY OF CALIFORNIA

MONTANA STATE UNIVERSITY

UNIVERSITY OF NEVADA

NEW MEXICO STATE UNIVERSITY

OREGON STATE UNIVERSITY

UNIVERSITY OF OREGON

OSAKA UNIVERSITY
UNIVERSITY OF SOUTHERN CALIFORNIA

STANFORD UNIVERSITY

UNIVERSITY OF TOKYO

UNIVERSITY OF UTAH

WASHINGTON STATE UNIVERSITY

UNIVERSITY OF WASHINGTON

AMERICAN MATHEMATICAL SOCIETY NAVAL WEAPONS CENTER 


\section{Pacific Journal of Mathematics}

\section{Vol. 55, No. $1 \quad$ September, 1974}

Robert Lee Anderson, Continuous spectra of a singular symmetric

differential operator on a Hilbert space of vector-valued functions . . . $\quad 1$

Michael James Cambern, The isometries of $L^{p}(X, K) \ldots \ldots \ldots \ldots \ldots . . \ldots$

R. H. Cameron and David Arne Storvick, Two related integrals over spaces of continuous functions ................................

Gary Theodore Chartrand and Albert David Polimeni, Ramsey theory and

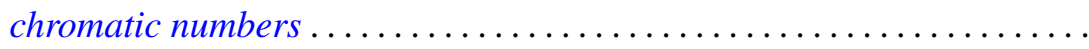

John Deryck De Pree and Harry Scott Klein, Characterization of collectively compact sets of linear operators ...................

John Deryck De Pree and Harry Scott Klein, Semi-groups and collectively compact sets of linear operators ....................... 55

George Epstein and Alfred Horn, Chain based lattices.............. 65

Paul Erdős and Ernst Gabor Straus, On the irrationality of certain series . . 85

Zdeněk Frolík, Measurable uniform spaces................... 93

Stephen Michael Gagola, Jr., Characters fully ramified over a normal

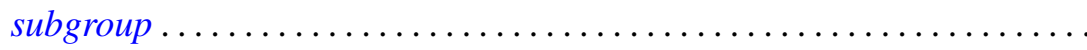

Frank Larkin Gilfeather, Operator valued roots of abelian analytic

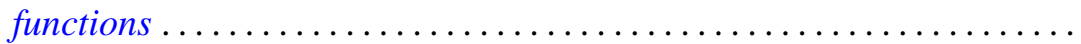

D. S. Goel, A. S. B. Holland, Cyril Nasim and B. N. Sahney, Best approximation by a saturation class of polynomial operators

James Secord Howland, Puiseux series for resonances at an embedded

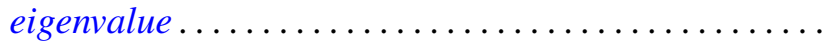

David Jacobson, Linear GCD equations .................

P. H. Karvellas, A note on compact semirings which are multiplicative

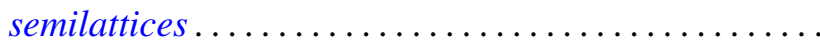

Allan Morton Krall, Stieltjes differential-boundary operators. II . .

D. G. Larman, On the inner aperture and intersections of convex sets

S. N. Mukhopadhyay, On the regularity of the $P^{n}$-integral and its application to summable trigonometric series ....... .

Dwight Webster Read, On $(J, M, m)$-extensions of Boolean algebras ....

David Francis Rearick, Multiplicativity-preserving arithmetic power series.

Indranand Sinha, Characteristic ideals in group algebras

Charles Thomas Tucker, II, Homomorphisms of Riesz spaces . . .

Kunio Yamagata, The exchange property and direct sums of indecomposable injective modules. 